\title{
MicroRNA 200b promotes mesenchymal-to-epithelial transition in anaplastic thyroid carcinoma
}

\author{
SHUNJI TAMAGAWA ${ }^{1}$, KEISUKE ENOMOTO ${ }^{1}$, ESRA GUNDUZ ${ }^{1}$, MEHMET GUNDUZ ${ }^{1}$, \\ FUYUKI SATO $^{2}$, SHINYA UCHINO ${ }^{3}$, YASUTERU MURAGAKI ${ }^{2}$ and MUNEKI HOTOMI ${ }^{1}$
}

\author{
Departments of ${ }^{1}$ Otolaryngology-Head and Neck Surgery and ${ }^{2}$ Pathology, Wakayama Medical University, Wakayama, \\ Wakayama 641-8509; ${ }^{3}$ Noguchi Thyroid Clinic and Hospital Foundation, Beppu, Oita 874-0902, Japan
}

Received September 20, 2019; Accepted May 27, 2020

DOI: 10.3892/ol.2020.11864

\begin{abstract}
Anaplastic thyroid cancer (ATC) remains a cancer with one of the worst prognoses, despite novel targeted therapies. The median survival rate has not improved for decades. Epithelial-to-mesenchymal transition (EMT) is a crucial step in physiological processes and in cancer progression, but the underlying mechanisms are not yet fully understood. The current study examined the role of microRNA (miR)-200b in mesenchymal-to-epithelial transition in ATC. Total RNA and miR isolation were performed from ATC cell lines transfected with a miR-200b mimic. After miR-200b mimic transfection, expression levels of E-cadherin, vimentin and zinc finger E-box binding homeobox 1 (ZEB1) were confirmed by reverse transcription-quantitative $\mathrm{PCR}$ and western blotting. Additionally, cell migration was evaluated using miR-200b mimic and scrambled negative control-transfected cells. A total of 14 human ATC and 15 non-cancerous human thyroid tissues were immunohistochemically stained and scored as controls for E-cadherin, vimentin and ZEB1. In ATC tissues and cell lines, the mesenchymal marker ZEB1 was significantly upregulated and the epithelial marker E-cadherin was significantly downregulated. Additionally, the mesenchymal marker vimentin was significantly upregulated in ATC tissues and in one ATC cell line. MiR-200b mimic transfection significantly increased vimentin and ZEB1 expression, but E-cadherin expression remained below the measurement sensitivity. Furthermore, miR-200b overexpression decreased cell migration. The current study suggested that miR-200b may regulate the expression levels of mesenchymal markers such as vimentin and ZEB1 in ATC and may promote mesenchymal-to-epithelial transition.
\end{abstract}

Correspondence to: Professor Muneki Hotomi, Department of Otolaryngology-Head and Neck Surgery, Wakayama Medical University, 811-1 Kimiidera, Wakayama, Wakayama 641-8509, Japan E-mail:mhotomi@wakayama-med.ac.jp

Key words: anaplastic thyroid carcinoma, epithelial-to-mesenchymal transition, microRNA 200b, vimentin, zinc finger E-box binding homeobox 1

\section{Introduction}

Anaplastic thyroid cancer (ATC) is a highly malignant undifferentiated subset of thyroid cancer (1). Although ATC represents $1-2 \%$ of thyroid cancer cases, patients with ATC diagnosed at the Mary Babb Randolph Cancer Center (Morgantown, USA) between 1987 and 2007 had a median survival of 3-5 months after diagnosis, due to its aggressiveness and the lack of effective treatment options (1). Despite informational and technological improvement in medical therapies, the median survival period in months for ATC has not improved. While some studies report that the incidence rates of ATC have decreased worldwide $(2,3)$, another study reports an increased incidence rate of ATC between 1973-2014 in the United States (4). For the treatment of ATC, it is preferable to resect the tumor and treat with external beam radiation for loco-regional control, followed by single or in-combination chemotherapy (3). Due to the challenging molecular complexity of ATC, targeted therapies are considered to be the most encouraging treatment modalities to improve prognosis in patients with ATC. However, novel targeted therapies tested over the last 10 years have not yet been approved for ATC. However, clinical trials are still ongoing and present promising results (2).

In addition to wound healing, epithelial-to-mesenchymal transition (EMT) is a crucial step in embryonic developmental processes, through which epithelial cells are converted to mesenchymal cells; EMT also occurs in cancer invasion and metastasis, so it is proposed as a promising target for cancer therapy (5). During EMT, a complex network of EMT transcription factors, including snail family transcriptional repressor 1 and 2 (SNAI1 and SNAI2), zinc finger E-box binding homeobox 1 and 2 (ZEB1 and ZEB2), and twist family bHLH transcription factor 1 and 2 (TWIST1 and TWIST2), are targeted to repress epithelial markers (such as E-cadherin) while activating mesenchymal markers (such as vimentin) via different levels of epigenetic, transcriptional and post-transcriptional regulations (5). Additionally, low E-cadherin expression is an important prognostic marker for ATC and is associated with an aggressive phenotype of thyroid cancer $(6,7)$.

ZEB1 is a zinc finger E-box binding protein that regulates the promoter of E-cadherin and serves a major role in 
EMT (8). In various types of cancer, such as oral cancer, and hepatocellular and renal carcinoma, ZEB1 upregulation has been identified as a prognostic factor (9-11). ZEB1 is regulated via numerous mechanisms, one of which acts via the microRNA (miR)-200 family; miR-200 is a tumor suppressor family with five members clustered as miR-200b/c/429 and miR-200a/141 based on their chromosomal region (12). These miRs are considered as determining factors of the epithelial phenotype of cancer cells via targeting the E-cadherin repressor ZEB1 (12).

The miR-200 family is involved in EMT, as well as in the repression, self-renewal and differentiation of cancer stem cells, modulation of cell division, apoptosis and the reversal of chemo-resistance (13). Therefore, numerous human cancer studies have focused on miR-200 (13). In a previous review, miR-200b was defined as an important regulator of EMT via regulating ZEB1 and ZEB2 in ATC (14). In the current study, the role of miR-200b on mesenchymal-to-epithelial transition was examined in ATC.

\section{Materials and methods}

Tissue collection. A total of 14 human ATC collected from 2003 to 2016 (from 5 male and 9 female patients; age range, 56-82 years; median age, 69 years) and 15 non-cancerous human thyroid tissues collected from 2014 to 2017 (from 2 male and 13 female individuals; age range, 43-83 years; median age, 65 years), namely adenomatous goiter (AG) tissues (used as controls), from different patients were obtained from the Noguchi Thyroid Clinic and Hospital Foundation (Beppu, Japan) in accordance with ethical approval from the Noguchi Thyroid Clinic and Hospital Foundation (approval no. 020) and Wakayama Medical University School of Medicine (approval no. 2449). All patients provided written informed consent for participation in the present study.

Immunohistochemicalstaining.Immunohistochemicalstaining was performed on $10 \%$ formalin-fixed paraffin-embedded tissue blocks using a Discovery Auto-Stainer with automated protocols (Ventana Medical Systems, Inc.). The primary antibodies used were anti-E-cadherin (1:400; cat. no. 3195) and anti-vimentin (1:200; cat. no. 5741) from Cell Signaling Technology, Inc., and anti-ZEB1 (1:100; cat. no. ab203829) from Abcam, in addition to the Discovery Universal secondary antibody (cat. no. 760-4205). Two clinical pathologists (FS and YM) independently scored the staining intensity in ATC and non-cancerous tissues using a light microscope (magnification, x40; Nikon Eclipse TS2; Nikon Corporation) for E-cadherin, vimentin and ZEB1 expression. Staining scores were recorded as follows: 0 , negative staining in the cell membrane; $1,<10 \%$ of weak staining in the cell membrane; $2,>10 \%$ of weak or moderate staining in the cell membrane; and $3,>50 \%$ of strong staining in the cell membrane (15).

Cell culture. The ATC 8505c, ASH-3 and KMH-2 cell lines were purchased from the Japanese Collection of Research Bioresources Cell Bank, and the normal human thyroid follicular epithelial Nthy-ori 3-1 cell line was purchased from Sigma-Aldrich (Merck KGaA). The 8505c cell line contained a C:G to G:C transversion at codon 248 of the p53 gene, and there were chromosomal abnormalities in the ASH-3 and KMH-2 cell lines at chromosome numbers 79-89 and 92-108, respectively.

The ATC and normal human thyroid follicular epithelial cell lines were grown in RPMI-1640 medium (Gibco; Thermo Fisher Scientific, Inc.) supplemented with 10\% FBS (HyClone; Cytiva), $1 \%$ penicillin/streptomycin and $1 \mu \mathrm{l} / \mathrm{ml}$ amphotericin B (Gibco; Thermo Fisher Scientific, Inc.). All cell lines were cultured in a humidified incubator at $37^{\circ} \mathrm{C}$ with $5 \% \mathrm{CO}_{2}$.

$m R N A$ processing and reverse transcription-quantitative $P C R(R T-q P C R)$. Preparation of cDNA from total RNA was performed directly from cultured cell lysates using the TaqMan Gene Expression Cells-to-CT kit (Ambion; Thermo Fisher Scientific, Inc.), according to the manufacturer's protocol. Briefly, cultured cells $\left(5 \times 10^{4}\right.$ cells/well; $70 \%$ confluence) in 96-well plates were washed with PBS and lysed with lysis buffer, and RNA was released into the supernatant. RNA was reverse transcribed into cDNA at $37^{\circ} \mathrm{C}$ for $60 \mathrm{~min}$ and $95^{\circ} \mathrm{C}$ for 5 min using the RT Enzyme mix and appropriate RT buffer (Ambion; Thermo Fisher Scientific, Inc.). Finally, cDNA was amplified by RT-qPCR using the TaqMan Gene Expression Master mix and the specific TaqMan primer/probe assay designed for the investigated genes: E-cadherin (accession no.Hs01023894_m1),vimentin(accessionno.Hs00185584_m1), ZEB1 (accession no. Hs00232783_m1) and GAPDH (accession no. Hs02758991_m1) (Applied Biosystems; Thermo Fisher Scientific, Inc.).

Gene expression levels were normalized to the expression levels of the housekeeping gene, GAPDH, and expressed as the relative fold change compared with the control sample. The amplification profile was initiated by $10 \mathrm{~min}$ of incubation at $95^{\circ} \mathrm{C}$, followed by a two-step amplification of $15 \mathrm{sec}$ at $95^{\circ} \mathrm{C}$ and $60 \mathrm{sec}$ at $60^{\circ} \mathrm{C}$ for 40 cycles, and a final extension step at $95^{\circ} \mathrm{C}$ for $5 \mathrm{~min}$. To exclude reagent contamination, all experiments were performed with non-template controls. Quantification was performed using the $2^{-\Delta \Delta C q}$ calculation method (16). Each sample was analyzed in triplicate on a 7500 Real-time PCR system in a 96-well plate (Applied Biosystems; Thermo Fisher Scientific, Inc.).

miRNA processing and $R T-q P C R$. Preparation of $\mathrm{cDNA}$ from miRNA was performed directly from cultured cell lysates using the TaqMan MicroRNA Cells-to-CT kit (Ambion; Thermo Fisher Scientific, Inc.) according to the manufacturer's protocol. Briefly, the cultured cells in 96-well plates were washed with PBS and lysed with lysis buffer, releasing the RNA into the supernatant. RNA was reverse transcribed into cDNA at $37^{\circ} \mathrm{C}$ for $60 \mathrm{~min}$, followed by $95^{\circ} \mathrm{C}$ for $5 \mathrm{~min}$ and $4^{\circ} \mathrm{C}$ until further use using the RT Enzyme mix, RT buffer and appropriate RT primer (Applied Biosystems; Thermo Fisher Scientific, Inc.). Finally, the cDNA of each mature miRNA was amplified with the same conditions as the mRNAs $\left(10 \mathrm{~min}\right.$ at $95^{\circ} \mathrm{C}$, followed by a two-step amplification of $15 \mathrm{sec}$ at $95^{\circ} \mathrm{C}$ and $60 \mathrm{sec}$ at $60^{\circ} \mathrm{C}$ for 40 cycles, and a final extension step at $95^{\circ} \mathrm{C}$ for $5 \mathrm{~min}$ ) using the TaqMan Universal Master mix and the specific primer and probe mix included in pre-designed TaqMan MicroRNA assays for hsa-miR-200b (cat. no. 4427975; Applied Biosystems; Thermo Fisher Scientific, Inc.). Quantification of miRNA expression 


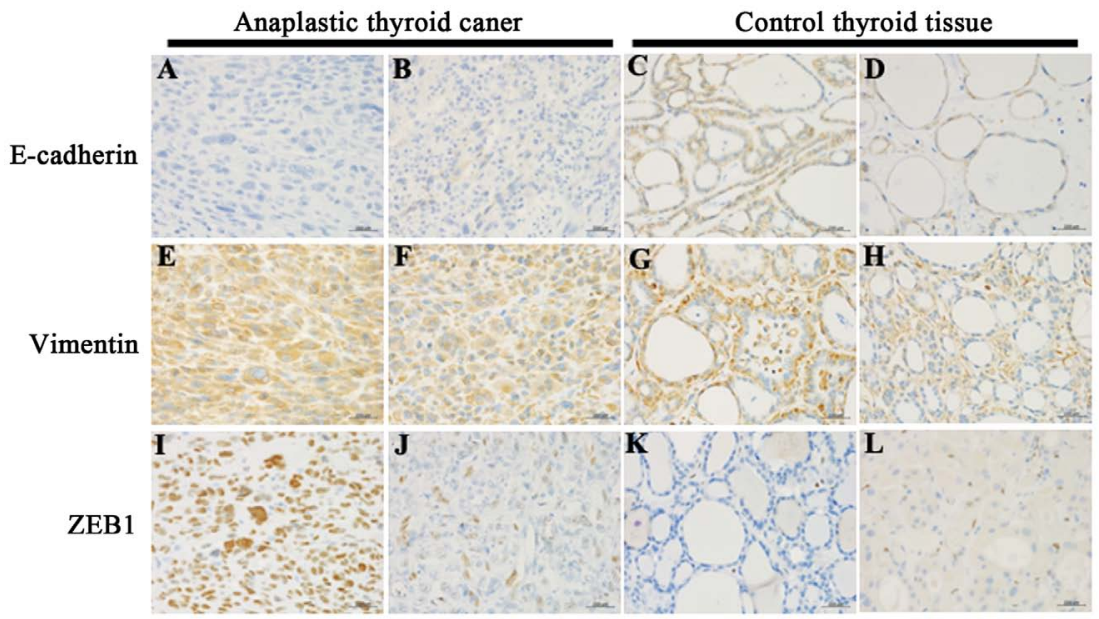

Figure 1. Immunohistochemical staining of E-cadherin, vimentin and ZEB1 in human ATC and control AG tissues. E-cadherin immunoreactivity scores of (A) 0 and (B) 1 in ATC tissues, and (C) 3 and (D) 2 in AG tissues. Vimentin immunoreactivity scores of (E) 3 and (F) 2 in ATC tissues, and (G) 3 and (H) 2 in AG tissues. ZEB1 immunoreactivity scores of (I) 3 and (J) 2 in ATC tissues, and (K) 0 and (L) 1 in AG tissues. Scale bar, $200 \mu$ m. ATC, anaplastic thyroid cancer; AG, adenomatous goiter; ZEB1, zinc finger E-box binding homeobox 1.

was performed using the aforementioned quantification method, and RNU6B RNA (cat. no. 001093; P/N 4373381; Applied Biosystems; Thermo Fisher Scientific, Inc.) was used as the internal control. miRNA levels were expressed as the fold change relative to the expression in untreated cells. Each sample was analyzed in triplicate.

Cell transfection with miR-200b mimic. The stabilityenhanced miR-200b mimic and the negative control, double-stranded RNA, were obtained from Applied Biosystems (Thermo Fisher Scientific, Inc.). Transfection of miRNA 200b mimic was carried out using Lipofectamine ${ }^{\circledR} 2000$ (Invitrogen; Thermo Fisher Scientific, Inc.) in accordance with the manufacturer's protocol. Briefly, the ASH-3 and KMH-2 cell lines ( $4 \times 10^{5}$ cells in $4 \mathrm{ml}$ RPMI-1640 with $10 \%$ FBS per plate) were seeded in 96-well culture plates and grown overnight in antibiotic-free medium before transfection. For western blot experiments, ASH- 3 and KMH- 2 cells $\left(1 \times 10^{6}\right.$ cells in $2 \mathrm{ml}$ of RPMI-1640 per dish) were seeded in 6-well culture dishes and grown overnight in antibiotic-free medium before transfection. The miR-200b-3p mimic (10 $\mu \mathrm{M} ; 5$ '-UAAUACUGCCUG GUAAUGAUGA-3') or the negative control (cat. no. 4464061; Thermo Fisher Scientific, Inc.) were transfected at the indicated concentrations into the cells using $0.3 \mu$ L Lipofectamine ${ }^{\circledR} 2000$ transfection reagent in $10 \mu \mathrm{l}$ Opti-MEM (Gibco; Thermo Fisher Scientific, Inc.) according to the manufacturer's protocol. The negative controls were scrambled oligonucleotides that were validated to not produce identifiable effects on known miRNA functions. miR-200b expression in the transfected cells was confirmed by RT-qPCR (using TaqMan MicroRNA assays) $24 \mathrm{~h}$ after transfection as aforementioned. Each sample was analyzed in triplicate.

Western blot analysis. Preparation of whole-cell lysates from human ATC cells has been previously described (15). Briefly, cultured cells were washed with PBS and homogenized in a solution containing $50 \mathrm{mM}$ Tris buffer, $150 \mathrm{mM} \mathrm{NaCl}, 1 \mathrm{mM}$ ethylene-diaminetetraacetic acid, 0.1\% Triton-X 100 (Sigma Aldrich; Merck KGaA), $1 \%$ NP-40 and proteinase/phosphatase inhibitors. Following centrifugation at $16,000 \mathrm{rpm}$ for $10 \mathrm{~min}$ at $4^{\circ} \mathrm{C}$, cell lysates were used for subsequent western blot analysis. Protein concentration was determined using the Pierce Coomassie Plus Protein assay kit (Thermo Fisher Scientific, Inc.) according to the manufacturer's protocol. Subsequently, proteins (20 $\mu \mathrm{g} /$ lane) were separated via 10\% SDS-PAGE, transferred to polyvinylidene fluoride membranes using Direct Blot (Gellex International), and immunostained using an automated iBind Western Device (Thermo Fisher Scientific, Inc.) at $24^{\circ} \mathrm{C}$, according to the manufacturer's protocol. The primary antibodies used were anti-vimentin (1:1,000; cat. no. 5741) and anti-ZEB1 (1:200; cat. no. 3396) from Cell Signaling Technology, Inc., and anti-GAPDH (1:2,000; cat. no. ab9484) from Abcam. The horseradish peroxidase-conjugated anti-mouse immunoglobulin G (IgG; 1:1,000; cat. no. 7076) or anti-rabbit $\operatorname{IgG}(1: 1,000$; cat. no. 7074) from Cell Signaling Technology, Inc., were used as the secondary antibodies, and subsequently detected using an enhanced chemiluminescence (ECL) system (Amersham ECL Prime; Cytiva). For control of protein loading, the blots were stripped using Re-Blot Plus (EMD Millipore) and re-probed with GAPDH. ImageJ64 software (v1.48; National Institutes of Health) was used for quantification of band intensities.

Wound healing assay. Wound healing assay was performed using the ATC ASH-3 and KMH-2 cell lines. The ATC cells were transfected separately with the miR-200b mimic or the scrambled negative control obtained from Ambion (Thermo Fisher Scientific, Inc.). Transfection was performed as aforementioned using Lipofectamine ${ }^{\circledR} 2000$ and Opti-MEM without FBS. When the cell confluence reached $\sim 80 \%$, wounds were created using a $200-\mu 1$ pipette tip and were then rinsed with RPMI-1640 medium to remove any free-floating cells and debris using the CytoSelect 24-well wound healing assay kit (Cell Biolabs, Inc). After $4 \mathrm{~h}$, the medium was replaced with fresh RPMI-1640 (with 10\% FBS), and after $8 \mathrm{~h}$ transfection was repeated, and after $4 \mathrm{~h}$, replacement with RPMI-1640 was repeated. Wound healing was observed at different time points $(0,12,36$ and $72 \mathrm{~h})$ within the scrape line, and representative 
images were captured using a light microscope at magnifications of $x 4$ and x10 (Nikon Eclipse TS2; Nikon Corporation). Nikon NIS-Element BR Analysis v5.11.00 software (Nikon Corporation) was used for the quantification of wound healing assays.

Statistical analysis. Data were analyzed using GraphPad Prism 5.0 (GraphPad Software, Inc.). To evaluate significant differences between two groups, the non-parametric Mann-Whitney $\mathrm{U}$ test was used. Data were presented as the mean $\pm \mathrm{SD}(\mathrm{n}=3)$. $\mathrm{P}<0.05$ was considered to indicate a statistically significant difference.

\section{Results}

Mesenchymal markers are upregulated in human ATC tissues. To investigate the EMT state in ATC tissues, the expression levels of E-cadherin, vimentin and ZEB1 were examined by immunohistochemical staining (Fig. 1). The patient clinicopathological characteristics and their immunohistochemical scores are summarized in Table I. Marked E-cadherin immunoreactivity in the cell membrane of cancer cells (score 2-3) was detected in non-cancerous control tissues $(100 \% ; 15 / 15)$, but not detected (score $0-1)$ in the ATC tissues $(0 \% ; 14 / 14)$. Vimentin immunoreactivity score of $\geq 2$ was detected in all ATC and normal or AG tissues (100\%; 14/14 and 15/15, respectively). Marked ZEB1 immunoreactivity scoring $\geq 2$ was observed in all ATC tissues $(100 \% ; 14 / 14)$, but not in AG tissues $(0 \% ; 0 / 15)$.

Mesenchymal markers are upregulated in ATC cell lines. To compare the expression levels of epithelial and mesenchymal markers at the RNA level, the normal thyroid follicular epithelium Nthy-ori 3-1 cell line and the undifferentiated thyroid carcinoma 8505c, ASH-3 and KMH-2 cell lines were used for RT-qPCR (Fig. 2). Compared with Nthy-ori 3-1 cells, E-cadherin expression was either not detected or very low in the ATC $8505 \mathrm{c}$, ASH-3 and KMH-2 cell lines, while vimentin expression was high in $8505 \mathrm{c}$ cells, but were similar to Nthy-ori 3-1 cells in ASH-3 and KMH-2 cell lines. The expression levels of the mesenchymal marker ZEB1 were high in all three ATC cell lines compared with Nthy-ori 3-1 cells (Fig. 2A). Furthermore, the ATC cell lines exhibited markedly decreased miR-200b expression compared with Nthy-ori 3-1 cells (Fig. 2B).

miR-200b regulates EMT markers in ATC cell lines. To further investigate the association between miR-200b and EMT markers (E-cadherin, vimentin and ZEB1), the effects miR-200b overexpression were analyzed on E-cadherin, vimentin and ZEB1 expression by transfecting the ASH-3 and KMH-2 cell lines with mimic miRNAs. Relative quantification was used to evaluate the expression levels of miR-200b following transfection with a negative control and with mimic miR-200b. Although no miR-200b expression was detected following transfection with the negative control, overexpression of miR-200b following transfection with the miRNA mimic was confirmed by RT-qPCR (data not shown).

miR-200b overexpression resulted in a significant downregulation of vimentin expression in $\mathrm{ASH}-3$ cells $(\mathrm{P}=0.028)$
Table I. Immunohistochemical expression of E-cadherin, vimentin and ZEB1 proteins in human ATC and control AG tissues.

\begin{tabular}{|c|c|c|c|c|c|c|}
\hline \multirow{2}{*}{$\begin{array}{l}\text { Case } \\
\text { no. }\end{array}$} & \multirow[b]{2}{*}{ Age } & \multirow[b]{2}{*}{ Sex } & \multirow[b]{2}{*}{ Histology } & \multicolumn{3}{|c|}{ Score $^{a}$} \\
\hline & & & & E-cadherin & Vimentin & ZEB1 \\
\hline 1 & 57 & $\mathrm{~F}$ & ATC & 0 & 3 & 3 \\
\hline 2 & 59 & $\mathrm{~F}$ & ATC & 0 & 3 & 3 \\
\hline 3 & 82 & $\mathrm{M}$ & ATC & 0 & 3 & 3 \\
\hline 4 & 61 & M & ATC & 0 & 3 & 2 \\
\hline 5 & 64 & $\mathrm{~F}$ & ATC & 0 & 3 & 2 \\
\hline 6 & 70 & $\mathrm{~F}$ & ATC & 0 & 3 & 3 \\
\hline 7 & 75 & $\mathrm{M}$ & ATC & 1 & 3 & 3 \\
\hline 8 & 78 & $\mathrm{~F}$ & ATC & 0 & 2 & 3 \\
\hline 9 & 69 & M & ATC & 0 & 3 & 2 \\
\hline 10 & 56 & $\mathrm{~F}$ & ATC & 0 & 3 & 2 \\
\hline 11 & 56 & $\mathrm{M}$ & ATC & 0 & 3 & 2 \\
\hline 12 & 74 & $\mathrm{~F}$ & ATC & 1 & 3 & 2 \\
\hline 13 & 69 & $\mathrm{~F}$ & ATC & 0 & 3 & 2 \\
\hline 14 & 77 & $\mathrm{~F}$ & ATC & 0 & 3 & 2 \\
\hline 15 & 82 & $\mathrm{~F}$ & $\mathrm{AG}$ & 3 & 3 & 0 \\
\hline 16 & 53 & $\mathrm{~F}$ & $\mathrm{AG}$ & 3 & 3 & 0 \\
\hline 17 & 53 & $\mathrm{M}$ & $\mathrm{AG}$ & 3 & 3 & 0 \\
\hline 18 & 66 & $\mathrm{~F}$ & $\mathrm{AG}$ & 3 & 2 & 0 \\
\hline 19 & 83 & $\mathrm{~F}$ & $\mathrm{AG}$ & 3 & 3 & 0 \\
\hline 20 & 65 & $\mathrm{~F}$ & $\mathrm{AG}$ & 3 & 3 & 1 \\
\hline 21 & 67 & $\mathrm{~F}$ & $\mathrm{AG}$ & 3 & 3 & 0 \\
\hline 22 & 46 & $\mathrm{~F}$ & $\mathrm{AG}$ & 3 & 3 & 0 \\
\hline 23 & 59 & $\mathrm{~F}$ & $\mathrm{AG}$ & 3 & 3 & 0 \\
\hline 24 & 73 & $\mathrm{~F}$ & $\mathrm{AG}$ & 2 & 3 & 0 \\
\hline 25 & 66 & $\mathrm{~F}$ & $\mathrm{AG}$ & 2 & 3 & 0 \\
\hline 26 & 64 & $\mathrm{~F}$ & $\mathrm{AG}$ & 3 & 3 & 0 \\
\hline 27 & 43 & $\mathrm{~F}$ & $\mathrm{AG}$ & 2 & 3 & 0 \\
\hline 28 & 81 & $\mathrm{~F}$ & $\mathrm{AG}$ & 3 & 3 & 0 \\
\hline 29 & 58 & $\mathrm{M}$ & $\mathrm{AG}$ & 3 & 3 & 0 \\
\hline
\end{tabular}

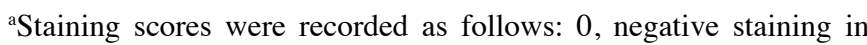
the cell membrane; $1,<10 \%$ of weak staining in the cell membrane; $2,>10 \%$ of weak or moderate staining in the cell membrane; and $3,>50 \%$ of strong staining in the cell membrane (15). ATC, anaplastic thyroid cancer; AG, adenomatous goiter; M, male; F, female; ZEB1, zinc finger E-box binding homeobox 1.

and a marked downregulation in $\mathrm{KMH}-2$ cells $(\mathrm{P}=0.200)$ compared with that in negative control-transfected cells (Fig. 3A). Similarly, ZEB1 expression was significantly downregulated in ASH-3 $(\mathrm{P}=0.028)$ and $\mathrm{KMH}-2$ cells $(\mathrm{P}=0.028)$ compared with negative control-transfected cells (Fig. 3B). E-cadherin expression remained below the measurement sensitivity, suggesting no direct association with miR-200b (data not shown). The present findings suggested that miR-200b promoted epithelial transition through suppression of ZEB1, which in turn repressed the mesenchymal marker vimentin in ATC cell lines.

Subsequently, changes in protein levels of EMT markers were evaluated via western blotting in ASH-3 and KMH-2 

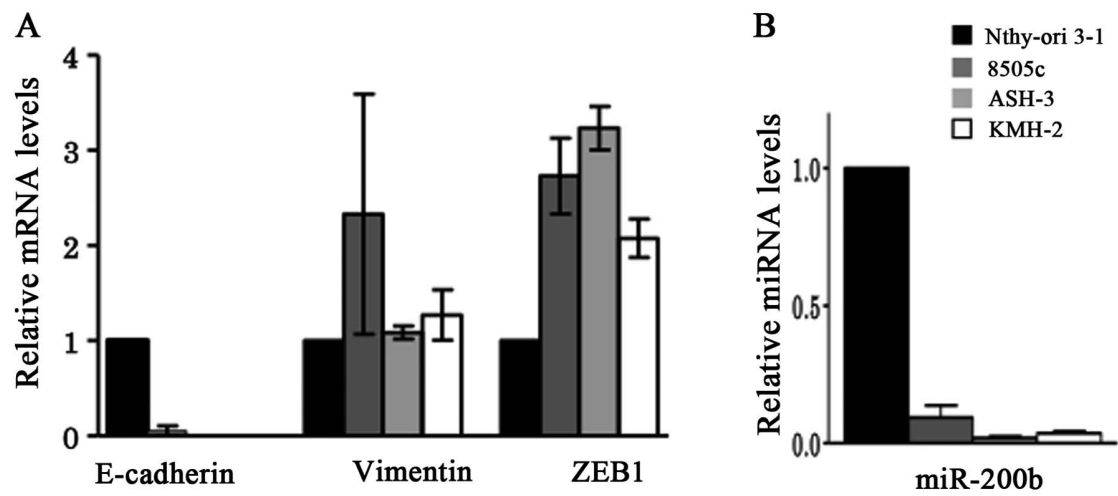

Figure 2. Relative mRNA and miRNA expression levels of epithelial-to-mesenchymal transition markers in thyroid cancer cells. mRNA expression levels of (A) E-cadherin, vimentin and ZEB1, and (B) miR-200b by reverse transcription-quantitative PCR in the anaplastic thyroid carcinoma 8505c, ASH-3 and KMH-2 cell lines, and the normal thyroid follicular epithelial Nthy-ori 3-1 cell line. ZEB1, zinc finger E-box binding homeobox 1; miRNA/miR-200b, microRNA-200b.

A

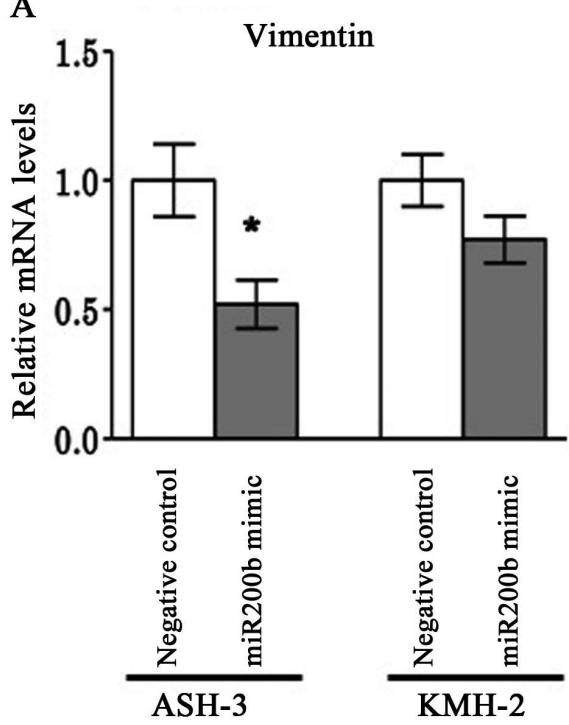

B

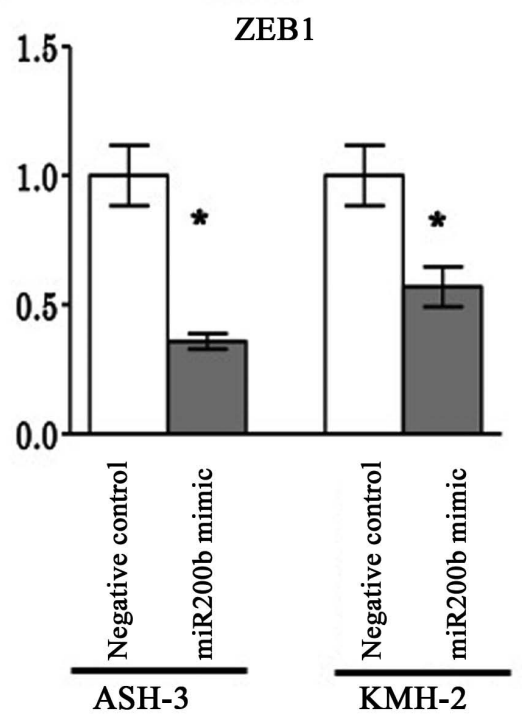

Figure 3. Relative mRNA levels of vimentin and ZEB1 in thyroid cancer cells. Changes in (A) vimentin and (B) ZEB1 mRNA expression following transfection with miR-200b mimic and negative control in ASH-3 and KMH-2 cell lines. The error bars represent the mean \pm standard error of the mean. ${ }^{*} \mathrm{P}<0.05$. ZEB1, zinc finger E-box binding homeobox 1; miR-200b, microRNA-200b.

cells following transfection with miR-200b mimic (Fig. 4). Similar to RT-qPCR results, compared with negative control-transfected cells, the protein levels of vimentin were significantly decreased in both KMH-2 and ASH-3 cells following transfection with the miR-200b mimic $(\mathrm{P}=0.030$ and $\mathrm{P}=0.031$, respectively). Furthermore, ZEB1 protein expression was significantly suppressed in both KMH-2 and ASH-3 cells $(\mathrm{P}<0.001$ and $\mathrm{P}=0.015$, respectively) compared with negative control-transfected cells. The present results suggested that miR-200b promoted mesenchymal-to-epithelial transition in human ATC cell lines.

miR-200b suppresses cell migration. Subsequently, the present study evaluated whether cell migration was associated with miR-200b. ASH-3 and KMH-2 cells were transfected with scrambled negative control and miR-200b mimic. Following transfection, miR-200b mimic-transfected cells migrated slower than the control cells, suggesting that wound healing was slower for cells with enforced miR-200b expression
(Fig. 5). Although 10\% FBS was used in the wound healing assays and a few cells were left in the middle of the wound during wound creation, which may have increased the proliferative activity and stimulated wound closure, the wound closure ratio was $90 \%$ in negative control cells and 70\% in ASH-3 cells with miR-200b overexpression after $36 \mathrm{~h}$ (Fig. 5A). In KMH-2 cells after $72 \mathrm{~h}$, wound closure was $60 \%$ for negative control cells and $40 \%$ for miR-200b mimic-transfected cells (Fig. 5B). The present findings suggested that miR-200b may reduce cell migration.

\section{Discussion}

EMT is considered to be an important phenomenon in invasion and metastasis. Whether genetically or epigenetically regulated, during EMT cancer cells first lose their epithelial properties and then gain mesenchymal characteristics and start migrating (17). Downregulated E-cadherin expression is a distinctive feature of EMT, resulting in loss of the epithelial 

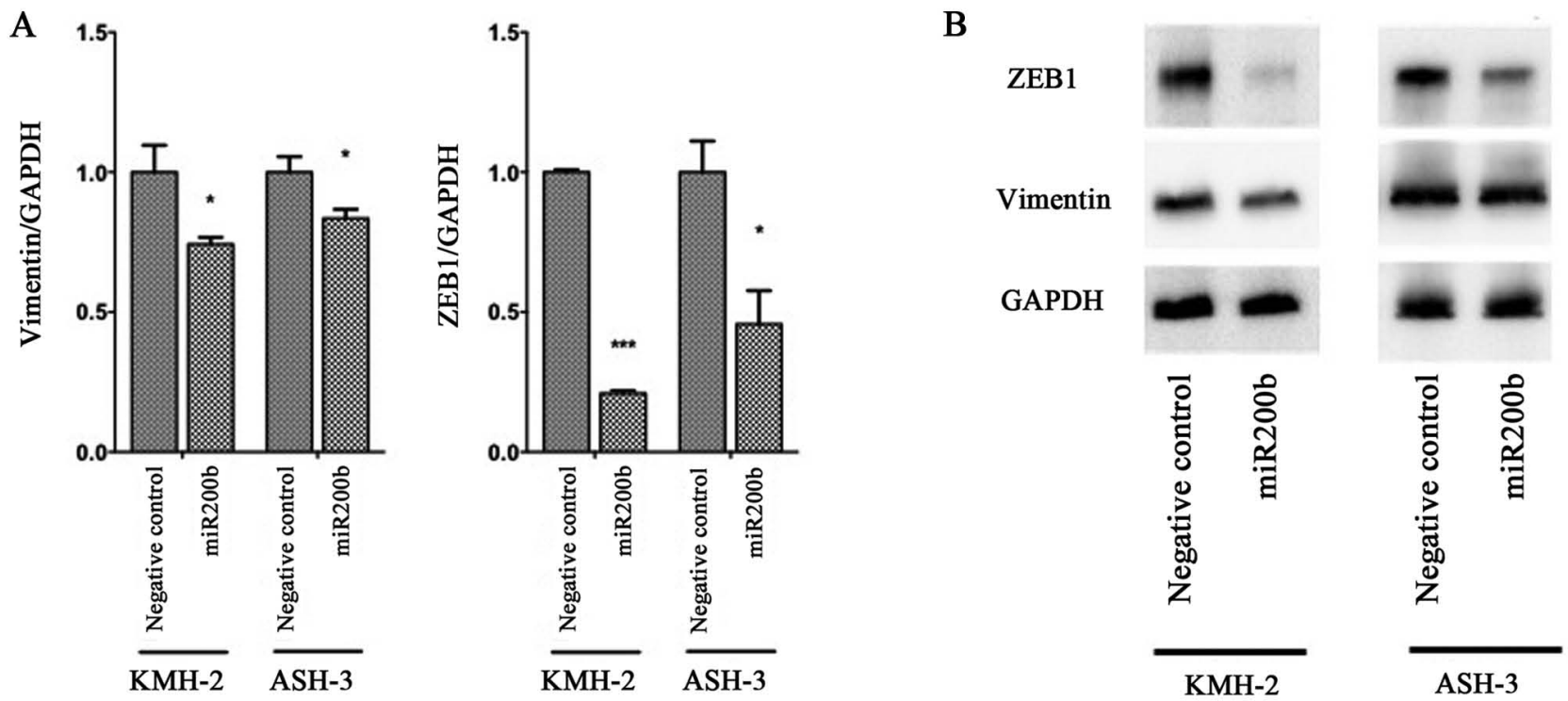

Figure 4. Western blot analysis of vimentin and ZEB1 protein expression following transfection with negative control and miR-200b mimic in ASH-3 and KMH-2 cells. (A) Quantification of (B) western blot bands. Error bars represent the mean \pm standard error of the mean. ${ }^{*} \mathrm{P}<0.05 ;{ }^{* * *} \mathrm{P}<0.001$. ZEB1, zinc finger E-box binding homeobox 1; miR-200b, microRNA-200b.
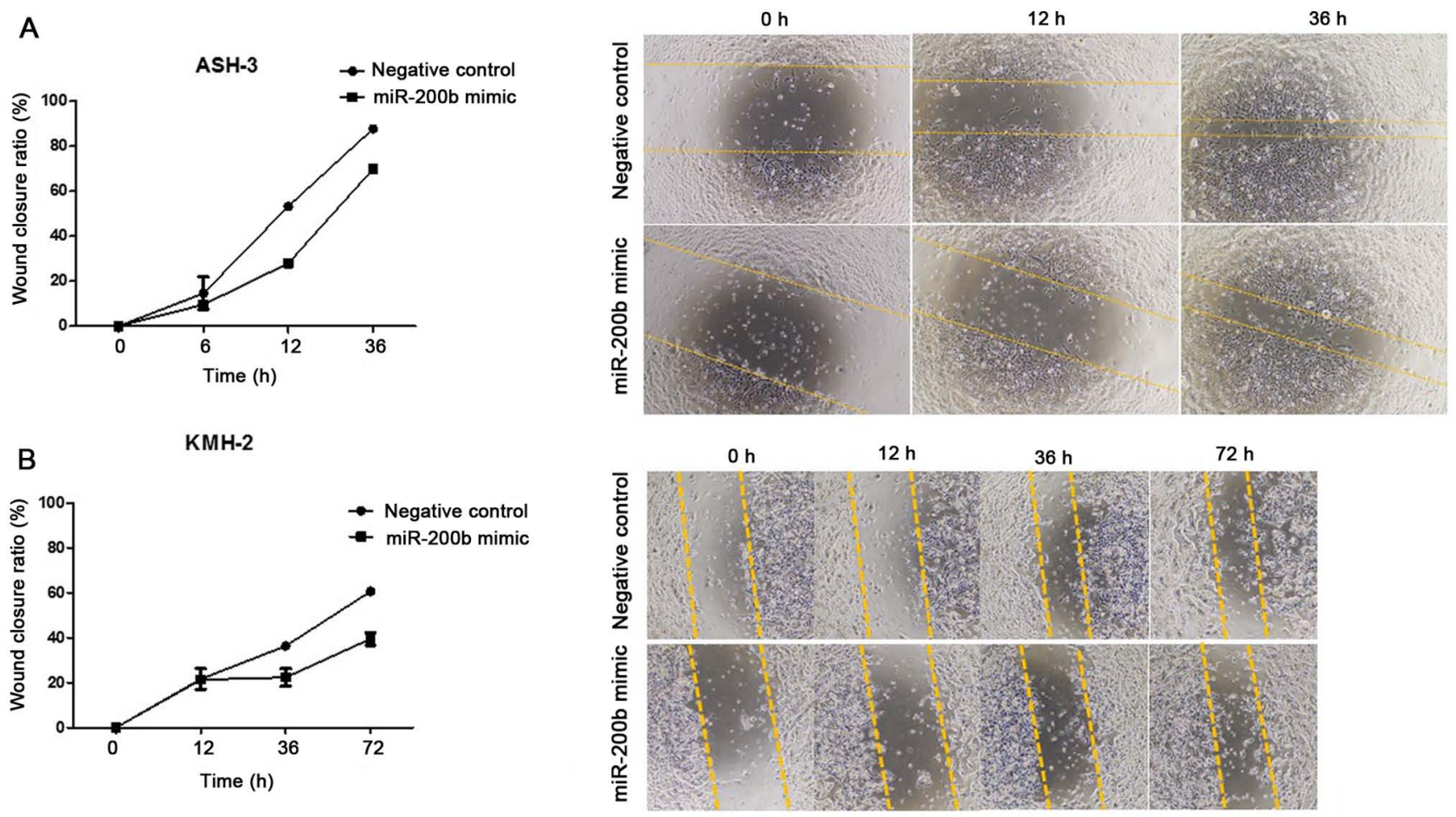

Figure 5. Wound healing assays in thyroid cancer cells. Representative images of wound healing assays of (A) ASH-3 and (B) KMH-2 cell lines after transfection with negative control and miR-200b mimic. miR-200b, microRNA-200b.

phenotype (17). In the current study, extremely low expression levels of E-cadherin were detected in ATC tissues compared with non-cancerous human thyroid tissues. Similarly, E-cadherin expression was not detected or was very low in the ATC 8505c, ASH-3 and KMH-2 cell lines, supporting the distinctive feature of EMT.

ZEB1 protein is thought to trigger the repression of epithelial genes while upregulating mesenchymal factors, leading to a dedifferentiation program (18). The findings of the present study revealed upregulated ZEB1 expression in ATC tissues and $8505 \mathrm{c}$, ASH-3 and KMH-2 cell lines compared with non-cancerous tissues and cells, respectively. Additionally, the expression levels of a representative mesenchymal marker, vimentin, did not differ between ATC and non-cancerous human thyroid tissues. Both ATC and AG tissues displayed high vimentin expression. Conversely, the results of vimentin expression in ATC cell lines exhibited varied expression levels: While expression in the ATC ASH-3 and KMH-2 cell lines 
did not differ from that in control cells, 8505c cells displayed higher vimentin expression compared with Nthy-ori 3-1 cells. In a previous study, vimentin expression was analyzed in ASH-3 and $\mathrm{KMH}-2$ cell lines, and although vimentin expression was detected, this was lower compared with the ATC IHH- 4 cell line, whereas the ATC TMH-1 cell line did not exhibit vimentin expression (19). This is in accordance with the present data revealing that while vimentin expression was high in $8505 \mathrm{c}$ cells, other cell lines, such as ASH-3 and KMH-2, displayed weaker vimentin expression. A potential explanation may be that vimentin is subject to epigenetic modifications in cancer (20). Additionally, vimentin is a biochemically diverse protein, and its diversity may be promoted by its size, assembly state, post-transcriptional modifications, co-assembly with other intermediate filaments and interactions with non-intermediate filaments (21).

ZEB and SNAI transcription factors, and members of the miR-200 and miR-34 families are involved in the EMT regulatory network (22). Additionally, in cells with an epithelial phenotype, there are high expression levels of miR-200 and miR-34, and low expression levels of ZEB and SNAI, while in cells with a mesenchymal phenotype there are high expression levels of ZEB and SNAI, and low expression levels of miR-200 and miR-34 (22). Loss of the miR-200 family is associated with aggressive cancer cell phenotypes, and downregulation of miR-200 leads to EMT, invasion and metastasis in cancer (23). Gregory et al reported that ZEB1 has five putative binding sites for miR-200b at the 3'-untranslated region, confirming previous data of ZEB1 being targeted by miR-200 family members (24). The present study revealed markedly decreased expression levels of miR-200b in ATC cell lines, and transfection with miR-200b mimic downregulated the mRNA expression levels of ZEB1 and vimentin in ASH-3 and KMH-2 cell lines. Additionally, the western blot results confirmed that the protein levels of ZEB1 and vimentin were also downregulated in ASH-3 and KMH-2 cell lines via enforced miR-200b expression, suggesting a potential role of miR-200b in EMT marker regulation.

miR-200 expression is decreased in ATC $(25,26)$. Zhang et al (26) demonstrated that epidermal growth factor $(\mathrm{EGF}) / \mathrm{EGF}$ receptor-induced EMT was regulated by the miR-200b family. As miR-200b restoration downregulated vimentin expression, the present results of the mesenchymal marker vimentin were similar to those reported by Zhang et al (26), although miR-200b restoration did not upregulate E-cadherin expression in the present study (data not shown). This may be due to various other mechanisms, such as methylation, that regulate E-cadherin expression (27). Although E-cadherin expression was not upregulated via miR-200b overexpression, the present results revealed that miR-200b overexpression decreased cell migration. The current data suggested an independent role of miR-200b from E-cadherin in cell migration.

The present study indicated that enforced miR-200b expression downregulated ZEB1 and vimentin expression, and suppressed cell migration in ATC cell lines. miR-200b may therefore promote mesenchymal-to-epithelial transition in ATC, and future studies may help to identify improved treatment modalities through the prevention of metastasis and cell invasion.

\section{Acknowledgements}

The authors would like to acknowledge proofreading and editing by Mr Benjamin Phillis at the Clinical Study Support Center of Wakayama Medical University (Wakayama, Japan).

\section{Funding}

The present study was partially supported by a Grant-in-Aid for Scientific Research (KAKENHI) from the Ministry of Education, Culture, Sports, Science and Technology of Japan (grant no. 18K16852).

\section{Availability of data and materials}

The datasets used and/or analyzed during the current study are available from the corresponding author on reasonable request.

\section{Authors' contributions}

ST, MG and MH designed the study. ST and KE acquired the data. ST, KE, FS, EG, MG, SU and YM analyzed the data. ST and EG prepared the manuscript. KE, FS, MG and SU edited the manuscript. $\mathrm{MH}$ controlled the quality of the data. YM and $\mathrm{MH}$ reviewed the manuscript. All authors read and approved the final manuscript.

\section{Ethics approval and consent to participate}

The present study received ethical approval from the Noguchi Thyroid Clinic and Hospital Foundation (grant no. 020) and Wakayama Medical University School of Medicine (grant no. 2449). All patients provided written informed consent to participate in the present study.

\section{Patient consent for publication}

Not applicable.

\section{Competing interests}

The authors declare that they have no competing interests.

\section{References}

1. Nagaiah G, Hossain A, Mooney CJ, Parmentier J and Remick SC: Anaplastic thyroid cancer: A review of epidemiology, pathogenesis, and treatment. J Oncol 2011: 542358, 2011.

2. Molinaro E, Romei C, Biagini A, Sabini E, Agate L, Mazzeo S, Materazzi G, Sellari-Franceschini S, Ribechini A, et al: Anaplastic thyroid carcinoma: From clinicopathology to genetics and advanced therapies. Nat Rev Endocrinol 13: 644-660, 2017.

3. Fagin JA and Wells SA Jr.: Biologic and Clinical Perspectives on Thyroid Cancer. N Engl J Med 375: 1054-1067, 2016.

4. Janz TA, Neskey DM, Nguyen SA and Lentsch EJ: Is the incidence of anaplastic thyroid cancer increasing: A population based epidemiology study. World J Otorhinolaryngol Head Neck Surg 5: 34-40, 2019.

5. Simeone P, Trerotola M, Franck J, Cardon T, Marchisio M, Fournier I, Salzet M, Maffia M and Vergara D: The multiverse nature of epithelial to mesenchymal transition. Semin Cancer Biol 58: 1-10, 2019. 
6. Tsiambas E, Ragos V, Georgakopoulos G, Rigopoulos DN Fotiades PP, Chatziioannidis A, Stamatelopoulos A, Vilaras G and Karameris A: E-cadherin/ $\alpha$-catenin deregulated co-expression in thyroid carcinoma based on tissue microarray digital image analysis. J Buon 21: 450-5, 2016.

7. Fourati A, El Amine O, Ben Ayoub W, Cherni I, Goucha A, El May MV, Gamoudi A and El May A: Expression profile of biomarkers altered in papillary and anaplastic thyroid carcinoma: Contribution of Tunisian patients. Bull Cancer 104: 433-441, 2017

8. Zhang Y, Xu L, Li A and Han X: The roles of ZEB1 in tumorigenic progression and epigenetic modifications. Biomed Pharmacother 110: 400-408, 2019.

9. Yao X, Sun S, Zhou X, Zhang Q, Guo $W$ and Zhang L: Clinicopathological significance of ZEB-1 and E-cadherin proteins in patients with oral cavity squamous cell carcinoma. Onco Targets Ther 10: 781-790, 2017.

10. Wan T, Zhang T, Si X and Zhou Y: Overexpression of EMT-inducing transcription factors as a potential poor prognostic factor for hepatocellular carcinoma in Asian populations: A meta-analysis. Oncotarget 8: 59500-59508, 2017.

11. Harb OA, Elfeky MA, El Shafaay BS, Taha HF, Osman G, Harera IS, Gertallah LM, Abdelmonem DM and Embaby A SPOP, ZEB-1 and E-cadherin expression in clear cell renal cell carcinoma (cc-RCC): Clinicopathological and prognostic significance. Pathophysiology 25: 335-345, 2018.

12. Park SM, Gaur AB, Lengyel E and Peter ME: The miR-200 family determines the epithelial phenotype of cancer cells by targeting the E-cadherin repressors ZEB1 and ZEB2. Genes Dev 22: 894-907, 2008.

13. Feng X, Wang Z and Fillmore R and Xi Y: miR-200, a new star miRNA in human cancer. Cancer Lett 344: 166-73, 2014.

14. Fuziwara CS and Kimura ET: MicroRNA deregulation in anaplastic thyroid cancer biology. Int J Endocrinol 2014: 743450, 2014

15. Enomoto K, Sato F, Tamagawa S, Gunduz M, Onoda N, Uchino S, Muragaki Y and Hotomi M: A novel therapeutic approach for anaplastic thyroid cancer through inhibition of LAT1. Sci Rep 9: 14616, 2019

16. Livak KJ and Schmittgen TD: Analysis of relative gene expression data using real-time quantitative PCR and the 2(-Delta C(T)) method. Methods 25: 402-8, 2001

17. Das V, Bhattacharya S, Chikkaputtaiah C, Hazra S and Pal M The basics of epithelial-mesenchymal transition (EMT): A study from a structure, dynamics, and functional perspective. J Cell Physiol, 2019 (Epub ahead of print).
18. Caramel J, Ligier M and Puisieux A: Pleiotropic Roles for ZEB1 in Cancer. Cancer Res 78: 30-35, 2018.

19. Sekiguchi M, Shiroko Y, Arai T, Kishino T, Sugawara I, Kusakabe T, Suzuki T, Yamashita T, Obara T, Ito K and Hasumi K: Biological characteristics and chemosensitivity profile of four human anaplastic thyroid carcinoma cell lines. Biomed Pharmacother 55: 466-74, 2001.

20. Satelli A and Li S: Vimentin in cancer and its potential as a molecular target for cancer therapy. Cell Mol Life Sci 68: 3033-3046, 2011.

21. Danielsson F, Peterson MK, Caldeira Araújo H, Lautenschläger F and Gad AKB: Vimentin diversity in health and disease. Cells 7: 147, 2018.

22. Garg M: Epithelial, mesenchymal and hybrid epithelial/mesenchymal phenotypes and their clinical relevance in cancer metastasis. Expert Rev Mol Med 19:e3, 2017.

23. Mongroo PS and Rustgi AK: The role of the miR-200 family in epithelial-mesenchymal transition. Cancer Biol Ther 10 219-222, 2010.

24. Gregory PA, Bert AG, Paterson EL, Barry SC, Tsykin A, Farshid G, Vadas MA, Khew-Goodall Y and Goodall GJ: The miR-200 family and miR-205 regulate epithelial to mesenchymal transition by targeting ZEB1 and SIP1. Nat Cell Biol 10: 593-601, 2008.

25. Braun J, Hoang-Vu C, Dralle $H$ and Hüttelmaier S: Downregulation of microRNAs directs the EMT and invasive potential of anaplastic thyroid carcinomas. Oncogene 29: 4237-44, 2010

26. Zhang Z, Liu ZB, Ren WM, Ye XG and Zhang YY: The miR-200 family regulates the epithelial-mesenchymal transition induced by EGF/EGFR in anaplastic thyroid cancer cells. Int $\mathbf{J}$ Mol Med 30: 856-62, 2012.

27. Jensen K, Patel A, Hoperia V, Larin A, Bauer A and Vasko V: Dynamic changes in E-cadherin gene promoter methylation during metastatic progression in papillary thyroid cancer. Ther Med 1: 457-462, 2010.

(i) $\Theta$ This work is licensed under a Creative Commons Attribution-NonCommercial-NoDerivatives 4.0 International (CC BY-NC-ND 4.0) License. 